\title{
Magnificação e ultrassom como recursos auxiliares no tratamento endodôntico em dentes com calcificação: considerações clínicas e relato de caso
}

Magnification and ultrasonics as auxiliary resources in endodontic treatment of calcified teeth: clinical considerations and case report

Magnificación y ultrasonido como recursos auxiliares en el tratamiento endodóntico en dientes con calcificación: consideraciones clínicas y reporte de caso

Ernani Canuto FIGUEIRÊDO JÚNIOR $\mathbf{~}^{1,3}$

Janaina Emanuella Galvão Menezes ALMEIDA² Eucaé Miranda MISSIAS ${ }^{3}$

Renata Correia Sotero Dália TORRES ${ }^{3}$

Mônica SOARES DE ALBUQUERQUE ${ }^{3,4}$

${ }^{1}$ Doutorando em Odontologia-Programa de Pós-Graduação em Odontologia, Universidade Estadual da Paraíba- UEPB 58429-500 Campina Grande - PB, Brasil

${ }^{2}$ Cirurgiã-Dentista, Especialista em Endodontia-Instituto de Odontologia da Paraíba-IOP, 58401-145, Campina Grande - PB, Brasil ${ }^{3}$ Instituto de Odontologia da Paraíba-IOP, 58401-145, Campina Grande - PB, Brasil

${ }^{4}$ Doutoranda em Odontologia - Programa de Pós-Graduação em Odontologia, Universidade de Pernambuco, 54756-220, Camaragibe-PE, Brasil

\section{Resumo}

O tratamento endodôntico tem como objetivo a limpeza, modelagem e obturação tridimensional dos canais radiculares Entretanto, a presença de alterações como calcificações podem trazer dificuldades, sendo necessário o uso de recursos auxiliares para a resolução de casos mais complexos. Assim, este trabalho tem como objetivo relatar um caso clínico do tratamento endodôntico em um dente que apresentava calcificações impedindo a localização de alguns canais radiculares, no qual foram utilizados recursos tecnológicos auxiliares como a tomografia computadorizada de feixe cônico, microscópio operatório e ultrassom. Além disso, foram feitas algumas considerações clínicas acerca da importância desses recursos na terapia endodôntica. Diante do presente caso clínico, o exame tomográfico constatou a presença de calcificação pulpar obliterando a entrada de alguns dos canais radiculares, sendo necessário o uso do ultrassom auxiliado pelo microscópio para remoção desta, a qual permitiu a realização do preparo químico-mecânico e da obturação dos canais, obtendo-se um desfecho satisfatório. Desse modo, pode-se concluir que diante de casos mais complexos, tais como aqueles em que há a presença de calcificações e de obliteração dificultando o acesso ao orifício de entrada dos canais radiculares, o uso destes recursos tecnológicos permite a realização do tratamento endodôntico com maior previsibilidade de sucesso.

Descritores: Endodontia; Tratamento do Canal Radicular; Calcificações da Polpa Dentária; Tomografia Computadorizada de Feixe Cônico; Microscopia; Ultrassom.

\section{Abstract}

Endodontic treatment aims at cleaning, shaping and three-dimensional filling of the root canals. However, the presence of alterations such as calcifications can cause difficulties, requiring the use of auxiliary resources to solve more complex cases. Thus, this work aims to report a clinical case of endodontic treatment on a tooth that presented calcifications precluding the location of some root canals, in which auxiliary technological resources were used, such as cone-beam computed tomography, microscopy and ultrasonic. In addition, some clinical considerations were made about the importance of these resources in endodontic therapy. In view of the present clinical case, the tomographic examination showed the presence of pulp calcification obliterating the entry of some of the root canals, requiring the use of ultrasonic aided by the microscope to remove it, which allowed the performance of the chemical-mechanical preparation and filling of the channels, obtaining a satisfactory result. Therefore, it can be concluded that in the face of more complex cases, such as those in which there is the presence of calcification and obliteration, making it difficult to access the root canal entry hole, the use of these technological resources allows endodontic treatment to be performed with greater predictability of success.

Descriptors: Endodontics; Root Canal Therapy; Dental Pulp Calcification; Cone-Beam Computed Tomography; Microscopy; Ultrasonics.

\section{Resumen}

El tratamiento endodóntico tiene como objetivo la limpieza, modelado y obturación tridimensional de los conductos radiculares Sin embargo, la presencia de cambios como las calcificaciones pueden causar dificultades, que requieren el uso de recursos auxiliares para resolver casos más complejos. Siendo así, el objetivo de este trabajo es informar un caso clínico de tratamiento endodóntico en un diente que presenta calcificaciones que impiden la ubicación de algunos conductos radiculares, en el que se utilizaron recursos tecnológicos auxiliares, como la tomografía computarizada de haz cónico, el microscopio operativo y el ultrasonido. Además, fueron hechas algunas consideraciones clínicas sobre la importancia de estos recursos en la terapia endodontica. En vista del presente caso clínico, el examen tomográfico mostró la presencia de calcificación pulpar obliterando la entrada de algunos de los conductos radiculares, lo que requería el uso de ultrasonido asistido por el microscopio para eliminarla, lo que permitió que se realizara la preparación químico-mecánica y la obturación de los canales, obteniendo un resultado satisfactorio. Por lo tanto, se puede concluir que en casos más complejos, como aquellos en que hay la presencia de calcificaciones y obliteraciones dificultando el acceso al orificio de entrada del conducto radicular, el uso de estes recursos tecnológicos permite la realización del tratamiento endodóntico con mayor previsibilidad de éxito.

Descriptores: Endodoncia; Tratamiento del Conducto Radicular; Calcificaciones de la Pulpa Dental; Tomografía Computarizada de Haz Cónico; Microscopía; Ultrasonido.

\section{INTRODUÇÃO}

Os objetivos do tratamento endodôntico (TE) consistem na limpeza, modelagem e preenchimento através da obturação tridimensional do sistema de canais radiculares (SCRs) $)^{1,2}$, estando o sucesso a longo prazo do TE intimamente associado a tais aspectos ${ }^{2,3}$. Entretanto, para a obtenção do sucesso etapas operatórias como a adequada realização da cirurgia de acesso e consequentemente a localização e identificação da abertura do canal radicular são requisitos importantes ${ }^{1}$.

Uma dificuldade que pode estar presente durante o TE consiste na obstrução do orifício da entrada dos canais radiculares ${ }^{1,3,4}$, o qual 
pode ocorrer em virtude da presença de estruturas calcificadas sob a forma de depósitos de dentina secundária ${ }^{1,4}$ ou de calcificações pulpares (CPs) propriamente ditas ${ }^{3}$.

A presença de estruturas calcificadas na polpa dental são alterações bastante comuns, podendo afetar dentes decíduos ou permanentes ${ }^{3}$. Enquanto estruturas calcificadas decorrentes da deposição de dentina secundária podem ocorrer em virtude da resposta à presença de material restaurador inserido próximo à polpa ${ }^{1}$, outras CPs podem ocorrer livremente na polpa ou estarem aderidas às paredes dentinárias, apresentando-se sob a forma de calcificações difusas ou de nódulos pulpares $(\mathrm{NPs})^{3,5}$, podendo os NPs apresentarem formato oval ou irregular de tamanho variável ${ }^{3,5}$.

Tanto a deposição de dentina secundária ${ }^{4}$ quanto as $\mathrm{CPs}^{3,5}$ podem obliterar a câmara pulpar. Consequentemente, tanto esta ${ }^{1,4}$, quanto as $\mathrm{CPs}^{3}$ podem obstruir os orifícios de entrada dos canais e dificultar a localização e o acesso ao SCRs ${ }^{1,4}$.

A tomografia computadorizada de feixe cônico (TCFC) tem se tornado uma ferramenta imprescindível na prática clínica endodôntica durante o diagnóstico e o planejamento do $\mathrm{TE}^{3-5}$, destacando-se como importante método auxiliar na avaliação da anatomia do $\mathrm{SCRs}^{3-5}$, bem como no diagnóstico de $\mathrm{CPs}^{3,5}$ e da localização de canais calcificados ${ }^{4,5}$.

Além disso, o uso de recursos auxiliares como o microscópio operatório (MO) promove uma magnificação com melhor iluminação e visualização do campo operatório, facilitando a localização dos canais radiculares calcificados e permitindo a execução do $\mathrm{TE}^{1}$. Somado a isso, o uso de dispositivos ultrassônicos permitem também auxiliar na localização dos canais radiculares calcificados e/ou de difícil acesso, sendo essas duas estratégias clínicas viáveis para acessar áreas de difícil acesso devido à presença de $\mathrm{CPs}^{1}$.

Diante disso, o objetivo deste estudo foi apresentar um relato de caso clinico do tratamento endodôntico utilizando microscópio e ultrassom em um molar permanente que apresentava calcificação na entrada de alguns dos seus condutos. Além disso, são feitas algumas considerações clínicas acerca da importância da utilização desses recursos tecnológicos para a realização do TE em casos complexos.

\section{CASO CLÍNICO}

Paciente de 44 anos de idade, do sexo feminino, saudável foi encaminhada para clínica de Especialização em Endodontia do Instituto de Odontologia da Paraíba-IOP, Campina Grande-PB para a realização de tratamento endodôntico do primeiro molar superior direito, com queixa de dor. Ao exame clínico extraoral não se constatou a presença de assimetria facial nem alteração em linfonodos. Já na avaliação clínica intrabucal, evidenciou-se que a paciente apresentava boa condição de saúde bucal, com restaurações dentárias preservadas, sendo que o dente 16 possuía extensa restauração em amálgama. Não se constou a presença de alterações nos tecidos bucais. Diante disso, foram realizados os testes térmicos e de percussão, obtendo-se em ambos respostas positivas. Frente à radiografia periapical inicial (Figura 1) observou-se ausência da imagem referente à câmara pulpar e ao canal disto-vestibular.

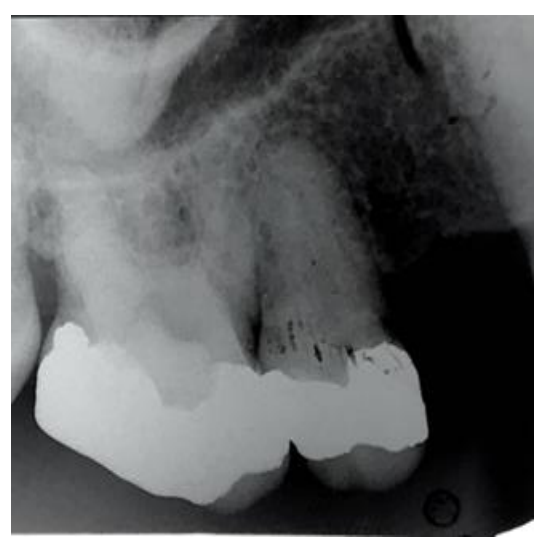

Figura 1: Radiografa periapical inicial. Observa-se ausência de imagem da câmara pulpar e dos canais mésio-vestibular e palatino, devido à presença de calcificação na região.

Com base nas informações coletadas durante a anamnese e nos achados clínicos e radiográficos, estabeleceu-se o diagnóstico de pulpite irreversível sintomática, realizando-se a seguir o TE. Assim, realizou-se anestesia local com lidocaína a $2 \%$ associada à epinefrina a 1:100.000 (Dentsply, Maillefer, Ballaigues, Suíça). Após abertura coronária foi realizado o isolamento absoluto com lençol de borracha e grampo, acompanhado de irrigação com hipoclorito de sódio a 2,5\%. Foi observado calcificação na região na entrada dos canais disto-vestibular e palatino, não sendo possível localizá-los. Além disso, constatou-se também a presença de atresia do canal mésio-vestibular. Assim, utilizou-se o microscópio operatório (Alliance, São Paulo, SP, Brasil) para obtenção de magnificação do campo operatório, visando auxiliar na condução do caso e realização do TE. A seguir realizou-se a irrigação abundante do canal mesio-vestibular utilizando-se solução de hipoclorito de sódio a 2,5\% e exploração com as limas manuais C Pilot \#06, \#08 e \#10 (VDW, Munich, Suíça) associadas ao EDTA (ácido 
Etilenodiaminotetracético) a 17\% (Biodinâmica, Ibiporã, PR, Brasil) para facilitar a instrumentação do mesmo e a localização dos demais canais radiculares. Entretanto não se conseguiu localizar o canal disto-vestibular e optou-se pela solicitação da realização de TCFC para pesquisa e avaliação da presença de calcificação e da localização do referido canal. Em seguida foi utilizada medicação intracanal através da aplicação de Otosporin® (FQM, Rio de Janeiro, RJ, Brasil) embebido em bolinha de espuma Pele Tim (Voco, Cuxhaven, Alemanha) estéril. Após isso, fez-se o selamento cavitário duplo utilizando-se cimento provisório coltosol (Coltene, Rio de Janeiro, RJ, Brasil) e cimento ionômero de vidro restaurador longlass (Maquira indústria de produtos odontológicos, Maringá, PR, Brasil). Na sessão seguinte, de posse do exame tomográfico (Figura 2), com o auxílio do $\mathrm{MO}$ e do ultrassom Jet Sonic BP (Gnatus, São Paulo, SP, Brasil), e dos insertos TRI27DP e TRI13 (Dental Trinks, São Paulo-SP, Brasil), removeu-se a calcificação do canal disto-vestibular e palatino, obtendo-se acesso direto a esses canais radiculares (Figura 3 ).

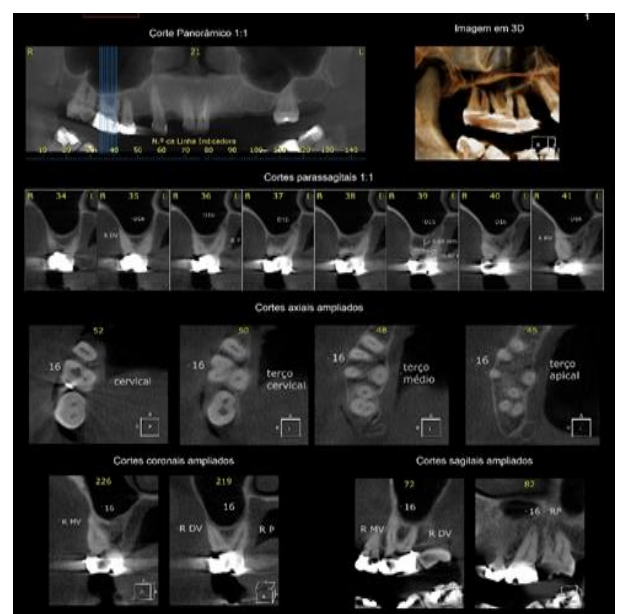

Figura 2: Imagens da tomografia computadorizada de feixe cônico

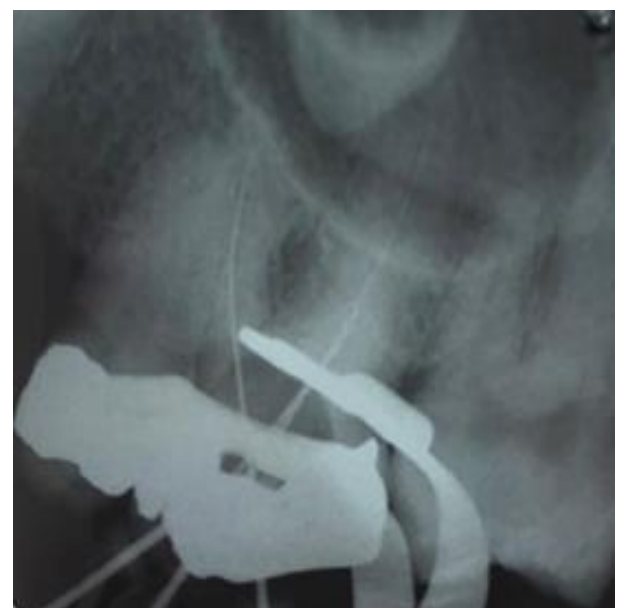

Figura 3: Radiografa periapical evidenciando-se o acesso aos canais radiculares após a remoção das calcificações

O preparo químico mecânico foi realizado com motor endodôntico Easy Enso SI
(Easy equipamentos odontológicos, Belo Horizonte, MG, Brasil), utilizando-se inicialmente a lima rotatória Prodesing Logic $® 15.03$ (Easy equipamentos odontológicos, Belo Horizonte, MG, Brasil) para glide path. O comprimento de trabalho foi determinado utilizando-se localizador apical eletrônico Propex Pixi (Dentsply, Maillefer, Ballaigues, Suíça). Em seguida, utilizaram-se limas reciprocantes Wave One Gold® (Dentsply, Maillefer, Ballaigues, Suíça) para a instrumentação dos canais, empregando-se as limas Small 20.07 e Primary 25.07 no canal disto-vestibular, enquanto que nos canais mésio-vestibular e palatino, além dessas mesmas limas, a instrumentação foi concluída com a lima Medium 35.06. Em todas as etapas da instrumentação, foram feitas irrigações abundantes com solução de hipoclorito de sódio a $2,5 \%$. Após a instrumentação, procedeu-se à seleção dos cones, sendo utilizados cones Odous de Deus tamanho FM (Odous de Deus, Belo Horizonte, MG, Brasil) devidamente calibrados de acordo com os tamanhos de ponta das limas empregadas na instrumentação, seguida de sua posterior confirmação através da realização de radiografia periapical. Em seguida, os cones foram desinfetados através de imersão em solução de hipoclorito de sódio a 2,5\%. Após essa etapa, o protocolo de limpeza final (toalete) foi realizado através da utilização de solução de hipoclorito de sódio a 2,5\%, seguida do EDTA 17\% (Biodinâmica, Ibiporã, PR, Brasil) e mais uma aplicação da solução de hipoclorito de sódio a $2,5 \%$, as quais foram agitadas com a lima Easy Clean (Easy equipamentos odontológicos, Belo Horizonte, MG, Brasil), em movimento reciprocante, empregando-se 3 ciclos de 20 segundos para cada uma das soluções. Em seguida, procedeu-se à secagem dos canais com pontas de papel absorvente estéril (Dentsply, Maillefer, Ballaigues, Suíça), de acordo com os respectivos tamanhos de ponta e conicidades empregados na instrumentação. A obturação foi realizada através da técnica do cone único e utilizou-se o cimento endodôntico AH Plus ${ }^{\circledR}$ (DentsplyDeTrey, Konstanz, Alemanha). Após inserção, corte e condensação dos cones, procedeu-se a limpeza da câmara pulpar utilizando-se escova para profilaxia da câmara pulpar (MKLife produtos médicos e odontológicos, Porto Alegre, $\mathrm{RS}$, Brasil) e gaze estéril embebida em álcool $70 \%$. Por fim, a blindagem coronária foi realizada através da colocação de cimento provisório coltosol (Coltene, Rio de Janeiro, RJ, Brasil) e preenchimento coronário com cimento ionômero de vidro restaurador longlass 
(Maquira indústria de produtos odontológicos, Maringá, PR, Brasil). A seguir realizou-se uma radiografia periapical final, observando-se adequada obturação endodôntica (Figura 4).

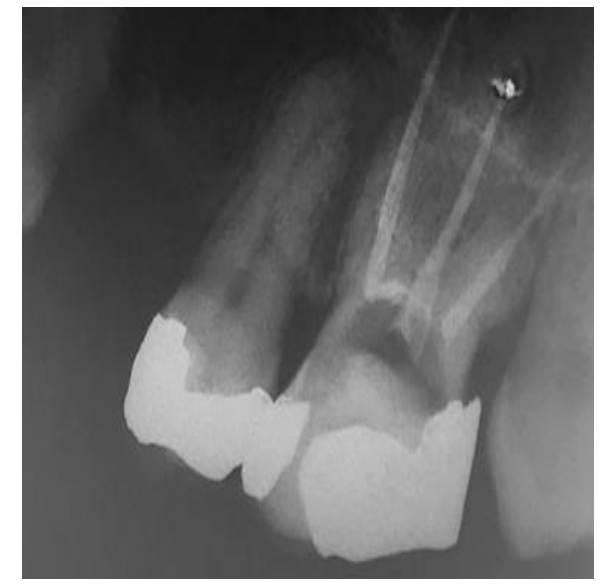

Figura 4: Radiografa final após obturação dos canais radiculares

DISCUSSÃO

Embora representem alterações comuns de etiologia incerta, os NPs são associados a fatores como infecção bacteriana, cárie profunda, presença de restaurações, doença periodontal, e envelhecimento ${ }^{3}$. Por outro lado, a deposição de dentina secundária calcificada pode ocorrer em virtude da presença de restaurações próximas à câmara pulpar ${ }^{1}$. No entanto, a despeito do tipo/origem da calcificação, tanto a presença de $\mathrm{NPs}^{3}$ quanto a presença de calcificação decorrente de depósitos dentinários ${ }^{1,4}$ são relatados como fatores que oferecem maior complexidade durante o TE.

Assim, uma das dificuldades presentes na prática clínica endodôntica é a localização de canais radiculares, sobretudo nos casos em que calcificações estão presentes na orifício de entradas dos canais e no assoalho da câmara pulpar conforme referido por Valdivia et al. ${ }^{1} \mathrm{e}$ observado no presente caso clínico.

Quanto à presença de calcificações, os achados referentes ao dente aqui apresentado permitem corroborar alguns dados da literatura, tal como a maior propensão de ocorrer calcificações em molares em virtude do maior tempo de exposição a processos degenerativos ou a agentes irritantes ${ }^{3}$. Por outro lado, a presença de outros tipos de calcificações como NPs também é atribuída predominantemente a esse grupo dentário ${ }^{5}$, sobretudo nos primeiros molares $^{3}$, mencionando-se ainda que a presença de restaurações aumenta a possibilidade de ocorrência dessas alterações ${ }^{5}$.

Diante dos aspectos apresentados, percebe-se que com a evolução tecnológica, o uso de estratégias e recursos tecnológicos como o $\mathrm{MO}$ e o uso do ultrassom facilitam a resolução de casos clínicos complexos, sendo tais ferramentas importantes para a obtenção do sucesso no $\mathrm{TE}^{2,4}$, permitindo a resolução de casos em que há a presença de calcificações na abertura de canais radiculares, conforme corroborado por Valdivia et al. ${ }^{1}$ e verificado no caso clínico anteriormente apresentado.

Além disso nessas condições, a TCFC destaca-se como um importante recurso imaginológico em virtude de sua eficiência para o diagnóstico de CPs e para a localização de canais radiculares ${ }^{3-5}$.

Nessas situações, a magnificação obtida a partir do $\mathrm{MO}$ é fundamental, sobretudo em procedimentos de localização e manejo de canais calcificados ${ }^{6,7}$. Assim, sua importância pode ser atribuída ao fato de ser um recurso auxiliar na detecção de diferenças entre a área calcificada e a dentina restante, tais como alterações na coloração ${ }^{1,2,6,7}$, a qual apresenta-se mais esbranquiçada nas áreas calcificadas $^{1}$, assim como mudanças texturais ${ }^{6}$. Além disso, a ampliação do campo de visão também facilita a localização dos canais radiculares e sua instrumentação ${ }^{1,2,6}$. Por outro lado, a utilização dos insertos ultrassônicos garantem um desgaste seletivo com segurança e controle, respeitando a anatomia dentária e sendo úteis na remoção de CPs e localização de canais radiculares calcificados, além de outras aplicações importantes em diferentes etapas do $\mathrm{TE}^{1}$.

Assim, diante da comparação no presente caso clínico entre a dificuldade inicial de localização e acesso aos canais radiculares e os resultados obtidos posteriormente ao uso do $\mathrm{MO}$ e do uso do ultrassom, corrobora-se que a utilização desses recursos tecnológicos na Endodontia fornecem maior facilidade operatória e segurança, oferecendo resultados satisfatórios e aumentando as chances da obtenção de sucesso do $\mathrm{TE}^{1,2,4}$.

CONCLUSÃO

Diante do exposto, percebe-se que em casos mais complexos durante o tratamento endodôntico de dentes que apresentam calcificações pulpares, o uso de estratégias e de aparatos tecnológicos como a tomografia computadorizada de feixe cônico para o diagnóstico e planejamento do caso clínico, a obtenção da magnificação através do emprego do microscópio operatório e o uso do ultrassom através de suas diversas aplicações são recursos essenciais, oferecendo resultados seguros e aumentando 0 índice de previsibilidade e sucesso da terapia endodôntica. 


\section{REFERÊNCIAS}

1. Valdivia JE, Pires M MP, Beltran HS, Machado MEL. Importance of ultrasound use in endodontic access of teeth with pulp calcification. Dental Press Endod. 2015; 5(2):67-73.

2. Iandolo $A$, landolo $G$, Malvano $M$, Pantaleo $G$, Simeone M. Modern technologies in Endodontics. Giornale Italiano di Endodonzia. 2016;30(1):2-9.

3. Hsieh CY, Wu YC, Su CC, Chung MP, Huang $R Y$, Ting $P Y$ et al. The prevalence and distribution of radiopaque, calcified pulp stones: A cone-beam computed tomography study in a northern Taiwanese population. J Dent Sci. 2018;13(2):138-44.

4. Lara-Mendes STO, Barbosa CFM, Santa-Rosa CC, Machado VC. Guided endodontic access in maxillary molars using cone-beam computed tomography and computer-aided design/computer-aided manufacturing system: a case report. J Endod. 2018;44(5):875-79.

5. da Silva EJNL, Prado MC, Queiroz PM, Nejaim Y, Brasil DM, Groppo FC, Haiter-Neto F. Assessing pulp stones by cone-beam computed tomography. Clin Oral Investig. 2017;21(7):2327-33.

6. Kim S, Baek S. The microscope and endodontics. Dent Clin North Am. 2004;48(1):11-8.

7. Feix LM, Boijink $D$, Ferreira $R$, Wagner $M H$, Barletta, FB. Microscópio operatório na Endodontia: magnificação visual e luminosidade. RSBO. 2010;7(3):340-48.

\section{CONFLITO DE INTERESSES}

Os autores declaram não haver conflitos de interesse

\section{AUTOR PARA CORRESPONDÊNCIA}

\section{Ernani Canuto Figueirêdo Júnior}

Universidade Estadual da Paraíba - UEPB

Departamento de Odontologia.

Rua das Baraúnas, 351, Bairro Universitário

58429-500 Campina Grande-PB, Brasil.

+ 55 (83) 3315-3300

E-mail: ernanicfjunior@outlook.com 